\title{
Ideals of direct products of rings
}

\author{
Ivan Chajda, Günther Eigenthaler and Helmut Länger
}

\begin{abstract}
It is known that an ideal of a direct product of commutative unitary rings is directly decomposable into ideals of the corresponding factors. We show that this does not hold in general for commutative rings and we find necessary and sufficient conditions for direct decomposability of ideals. For varieties of commutative rings we derive a Mal'cev type condition characterizing direct decomposability of ideals and we determine explicitly all varieties satisfying this condition.
\end{abstract}

\section{AMS Subject Classification: 13A15, 16R40, 08B05}

Keywords: Commutative ring, ring ideal, direct product, directly decomposable ideal, Mal'cev condition, variety of commutative rings

Recall that an ideal of a ring $\mathbf{R}=(R,+, \cdot)$ is a non-empty subset $I$ of $R$ such that if $a, b \in I$ then $a-b \in I$ and $a r, r a \in I$ for every $r \in R$. For other concepts used here the reader is referred to any monograph on rings, see e.g. [1] and [4]. Ideals play a crucial role in the theory of rings since the kernels of homomorphisms are ideals and rings can be factorized by means of ideals.

It is an elementary fact that in rings, ideals and congruences are in a one-to-one correspondence. Hence, if $\mathbf{R}=(R,+, \cdot)$ is a ring then the ideal lattice $\mathbf{I d} \mathbf{R}:=(\operatorname{Id} \mathbf{R}, \subseteq)$ and the congruence lattice $\operatorname{Con} \mathbf{R}:=(\operatorname{Con} \mathbf{R}, \subseteq)$ are isomorphic. Hence $\mathbf{I d} \mathbf{R}$ is a modular bounded lattice with the least element $\{0\}$ and the greatest element $R$ where the supremum and infimum of ideals $I_{1}, I_{2}$ are given by $I_{1} \vee I_{2}=I_{1}+I_{2}$ and $I_{1} \wedge I_{2}=I_{1} \cap I_{2}$, respectively.

Having two rings $\mathbf{R}_{1}=\left(R_{1},+, \cdot\right)$ and $\mathbf{R}_{2}=\left(R_{2},+, \cdot\right)$, it is elementary that for $I_{1} \in \operatorname{Id} \mathbf{R}_{1}$ and $I_{2} \in \operatorname{Id} \mathbf{R}_{2}$ we have $I_{1} \times I_{2} \in \operatorname{Id}\left(\mathbf{R}_{1} \times \mathbf{R}_{2}\right)$. On the other hand, if $I \in \operatorname{Id}\left(\mathbf{R}_{1} \times \mathbf{R}_{2}\right)$ then there need not exist $I_{1} \in \operatorname{Id} \mathbf{R}_{1}$ and $I_{2} \in \operatorname{Id} \mathbf{R}_{2}$ with $I_{1} \times I_{2}=I$. If such ideals $I_{1}, I_{2}$ do not exist, then $I$ is called skew. Otherwise, $I$ will be called directly decomposable. For commutative rings $\mathbf{R}_{1}, \mathbf{R}_{2}$ we will derive conditions under which $\mathbf{R}_{1} \times \mathbf{R}_{2}$ has no skew ideals.

We say that a direct product of finitely many rings has directly decomposable ideals if every ideal of this product is a direct product of ideals of the corresponding factors.

For sets $M_{1}, M_{2}$ and $i \in\{1,2\}$ let $\pi_{i}$ denote the $i$-th projection from $M_{1} \times M_{2}$ onto $M_{i}$.

\footnotetext{
${ }^{1}$ Support of the research of all three authors by ÖAD, project CZ 04/2017, support of the first and the third author by IGA, project PřF 2018 012, as well as support of the second and the third author by the Austrian Science Fund (FWF), project I 1923-N25, is gratefully acknowledged.

${ }^{2}$ Electronic version of an article published in Asian-European Journal of Mathematics, Vol. 11, No. 4, 2018, pages 1850094-1-1850094-6, DOI: 10.1142/S1793557118500948 @ World Scientific Publishing Company, https://www.worldscientific.com/worldscinet/aejm
} 
It is easy to see that an ideal $I$ of $\mathbf{R}_{1} \times \mathbf{R}_{2}$ is directly decomposable if and only if $\pi_{1}(I) \times \pi_{2}(I)=I$ which is equivalent to $\pi_{1}(I) \times \pi_{2}(I) \subseteq I$.

Direct decomposability of ideals in commutative rings was used by the first and the third author in their study of complementation in ideal lattices, see [2].

Let $\mathbf{K}_{4}=(\{0, a, b, c\},+)$ denote the four-element Kleinian group whose operation table for + looks as follows:

\begin{tabular}{c|cccc}
+ & 0 & $a$ & $b$ & $c$ \\
\hline 0 & 0 & $a$ & $b$ & $c$ \\
$a$ & $a$ & 0 & $c$ & $b$ \\
$b$ & $b$ & $c$ & 0 & $a$ \\
$c$ & $c$ & $b$ & $a$ & 0
\end{tabular}

We show an example of rings whose direct product contains a skew ideal.

Example 1. Consider the zero-ring $\mathbf{K}=(K,+, \cdot)$ whose additive group is $\mathbf{K}_{4}$, i.e., $x y=0$ for all $x, y \in K$. Then the principal ideal $I(a, c)=\{(0,0),(a, c)\}$ of $\mathbf{K} \times \mathbf{K}$ generated by $(a, c)$ is skew since $I \neq \pi_{1}(I) \times \pi_{2}(I)$.

In what follows, we derive necessary and sufficient conditions under which an ideal of the direct product of rings is directly decomposable. For this purpose, we borrow the method developed by Fraser and Horn ([3]) for congruences.

Theorem 2. Let $\mathbf{R}_{1}=\left(R_{1},+, \cdot\right)$ and $\mathbf{R}_{2}=\left(R_{2},+, \cdot\right)$ be rings and $I \in \operatorname{Id}\left(\mathbf{R}_{1} \times \mathbf{R}_{2}\right)$. Then the following are equivalent:

(i) I is directly decomposable.

(ii) $\left(R_{1} \times\{0\}\right) \cap\left(\left(\{0\} \times R_{2}\right)+I\right) \subseteq I$ and $\left(\left(R_{1} \times\{0\}\right)+I\right) \cap\left(\{0\} \times R_{2}\right) \subseteq I$.

(iii) If $(a, b) \in I$ then $(a, 0),(0, b) \in I$.

(iv) $\left(\left(R_{1} \times\{0\}\right)+I\right) \cap\left(\left(\{0\} \times R_{2}\right)+I\right)=I$.

Proof.

(i) $\Rightarrow$ (ii):

If $I=I_{1} \times I_{2}$ then

$$
\begin{aligned}
\left(R_{1} \times\{0\}\right) \cap\left(\left(\{0\} \times R_{2}\right)+I\right) & =\left(R_{1} \times\{0\}\right) \cap\left(\left(\{0\} \times R_{2}\right)+\left(I_{1} \times I_{2}\right)\right)= \\
& =\left(R_{1} \times\{0\}\right) \cap\left(I_{1} \times R_{2}\right)=I_{1} \times\{0\} \subseteq I, \\
\left(\left(R_{1} \times\{0\}\right)+I\right) \cap\left(\{0\} \times R_{2}\right) & =\left(\left(R_{1} \times\{0\}\right)+\left(I_{1} \times I_{2}\right)\right) \cap\left(\{0\} \times R_{2}\right)= \\
& =\left(R_{1} \times I_{2}\right) \cap\left(\{0\} \times R_{2}\right)=\{0\} \times I_{2} \subseteq I .
\end{aligned}
$$

(ii) $\Rightarrow$ (iii):

If $(a, b) \in I$ then

$$
\begin{aligned}
& (a, 0) \in\left(R_{1} \times\{0\}\right) \cap\left(\left(\{0\} \times R_{2}\right)+I\right) \subseteq I, \\
& (0, b) \in\left(\left(R_{1} \times\{0\}\right)+I\right) \cap\left(\{0\} \times R_{2}\right) \subseteq I .
\end{aligned}
$$

(iii) $\Rightarrow(\mathrm{i})$ :

If $(a, b) \in \pi_{1}(I) \times \pi_{2}(I)$ then there exists some $(c, d) \in R_{1} \times R_{2}$ with $(a, d),(c, b) \in I$, 
hence $(a, 0),(0, b) \in I$ which shows $(a, b)=(a, 0)+(0, b) \in I$.

(i) $\Rightarrow$ (iv):

If $I=I_{1} \times I_{2}$ then

$$
\begin{aligned}
& \left(\left(R_{1} \times\{0\}\right)+I\right) \cap\left(\left(\{0\} \times R_{2}\right)+I\right)= \\
& =\left(\left(R_{1} \times\{0\}\right)+\left(I_{1} \times I_{2}\right)\right) \cap\left(\left(\{0\} \times R_{2}\right)+\left(I_{1} \times I_{2}\right)\right)=\left(R_{1} \times I_{2}\right) \cap\left(I_{1} \times R_{2}\right)= \\
& =I_{1} \times I_{2}=I .
\end{aligned}
$$

(iv) $\Rightarrow$ (ii):

This follows immediately.

The following result is already known but it follows easily from the equivalence of (i) and (iii) in Theorem 2 .

Corollary 3. If $\mathbf{R}_{1}$ and $\mathbf{R}_{2}$ are unitary rings then $I$ is directly decomposable since $(a, b) \in$ $I$ implies $(a, 0)=(a, b)(1,0) \in I$ and $(0, b)=(a, b)(0,1) \in I$ and hence (iii) holds.

Corollary 4. Let $\mathbf{R}_{1}, \mathbf{R}_{2}$ be rings. Then $\mathbf{I d}\left(\mathbf{R}_{1} \times \mathbf{R}_{2}\right)$ is distributive if and only if every ideal of $\mathbf{R}_{1} \times \mathbf{R}_{2}$ is directly decomposable and $\mathbf{I} \mathbf{d} \mathbf{R}_{1}, \mathbf{I} \mathbf{d} \mathbf{R}_{2}$ are distributive.

Proof. If $\mathbf{I d}\left(\mathbf{R}_{1} \times \mathbf{R}_{2}\right)$ is distributive, then (ii) of Theorem 2 is satisfied for every $I \in$ $\operatorname{Id}\left(\mathbf{R}_{1} \times \mathbf{R}_{2}\right)$. Thus every ideal of $\mathbf{R}_{1} \times \mathbf{R}_{2}$ is directly decomposable. Moreover, for $i=1,2$ we have that $\mathbf{I d} \mathbf{R}_{i}$ is isomorphic to the principal filter of $\mathbf{I d}\left(\mathbf{R}_{1} \times \mathbf{R}_{2}\right)$ generated by the kernel of $\pi_{i}$. Therefore, $\mathbf{I} \mathbf{d} \mathbf{R}_{1}$ and $\mathbf{I} \mathbf{d} \mathbf{R}_{2}$ are distributive. Conversely, suppose that $\mathbf{I} \mathbf{d} \mathbf{R}_{1}$, Id $\mathbf{R}_{2}$ are distributive and every ideal of $\mathbf{R}_{1} \times \mathbf{R}_{2}$ is directly decomposable. Let $I, J \in \operatorname{Id}\left(\mathbf{R}_{1} \times \mathbf{R}_{2}\right)$. Then there exist $I_{1}, J_{1} \in \operatorname{Id} \mathbf{R}_{1}$ and $I_{2}, J_{2} \in \operatorname{Id} \mathbf{R}_{2}$ with $I_{1} \times I_{2}=I$ and $J_{1} \times J_{2}=J$. Now we have

$$
\begin{aligned}
& I \vee J=\left(I_{1} \times I_{2}\right)+\left(J_{1} \times J_{2}\right)=\left(I_{1}+J_{1}\right) \times\left(I_{2}+J_{2}\right), \\
& I \wedge J=\left(I_{1} \times I_{2}\right) \cap\left(J_{1} \times J_{2}\right)=\left(I_{1} \cap J_{1}\right) \times\left(I_{2} \cap J_{2}\right) .
\end{aligned}
$$

Hence, join and meet in $\mathbf{I d}\left(\mathbf{R}_{1} \times \mathbf{R}_{2}\right)$ are computed "component-wise" showing distributivity of $\mathbf{I d}\left(\mathbf{R}_{1} \times \mathbf{R}_{2}\right)$.

Another application of Theorem 2 is the following example:

Example 5. If $\mathbf{R}_{1}=\left(R_{1},+, \cdot\right)$ is a Boolean ring (i.e., $x x \approx x$ ) and $\mathbf{R}_{2}=\left(R_{2},+, \cdot\right)$ a unitary ring then $\mathbf{R}_{1} \times \mathbf{R}_{2}$ has no skew ideals. This follows directly by (iii) of Theorem $\mathbf{Q}$ since if $(a, b) \in I$ then $(a, 0)=(a, b)(a, 0) \in I$ and $(0, b)=(a, b)(0,1) \in I$.

Denote by $\mathbb{Z}$ the ring of integers. As usually, for $a \in \mathbb{Z}$ put $a \mathbb{Z}:=\{a x \mid x \in \mathbb{Z}\}$. Of course, for each $a \in \mathbb{Z}, a \mathbb{Z}$ is a commutative ring which is unitary only in case $a \in\{-1,0,1\}$. The next theorem shows that, in general, the rings $a \mathbb{Z} \times b \mathbb{Z}$ contain skew ideals. Hence, the rather exotic ring $\mathbf{K} \times \mathbf{K}$ from Example 1 is not the only commutative ring possessing skew ideals.

Theorem 6. Let $a, b, c, d \in \mathbb{Z}$ with $c \mid a$ and $d \mid b$ and consider the ideal $I(a, b)$ of $c \mathbb{Z} \times d \mathbb{Z}$ generated by $(a, b)$. Then $I(a, b)$ is directly decomposable if and only if either $a=0$ or $b=0$ or $\operatorname{gcd}(c, d)=1$. 
Proof. Put $(a, b) \mathbb{Z}:=\{(a x, b x) \mid x \in \mathbb{Z}\}$. Obviously, $I(a, b)=(a, b) \mathbb{Z}+(a c \mathbb{Z} \times b d \mathbb{Z})$. Since $\pi_{1}(I(a, b))=a \mathbb{Z}$ and $\pi_{2}(I(a, b))=b \mathbb{Z}$, direct decomposability of $I(a, b)$ is equivalent to $a \mathbb{Z} \times b \mathbb{Z} \subseteq I(a, b)$. If $a=0$ or $b=0$ then obviously $a \mathbb{Z} \times b \mathbb{Z} \subseteq I(a, b)$. Now assume $a, b \neq 0$. If $\operatorname{gcd}(c, d)=1$ then there exist $e, f \in \mathbb{Z}$ with $c e+d f=1$ and hence

$$
(a x, b y)=(a, b)(d f x+c e y)+((a c)(e(x-y)),(b d)(-f(x-y))) \in I(a, b)
$$

for all $x, y \in \mathbb{Z}$ proving direct decomposability of $I(a, b)$. Finally, assume $\operatorname{gcd}(c, d) \neq 1$. Suppose $I(a, b)$ to be directly decomposable. Then $(a, 0) \in I(a, b)$ and hence there exist $g, h, i \in \mathbb{Z}$ with $(a, 0)=(a g+a c h, b g+b d i)$. From this we conclude $g=-d i$ and hence $c h-d i=1$ whence $\operatorname{gcd}(c, d)=1$, a contradiction. Hence $I(a, b)$ is not directly decomposable in this case.

Although the rings of the form $c \mathbb{Z}$ are rings of integers, Theorem 6 yields the following result.

Corollary 7. If $c, d \in \mathbb{Z}$ and $\operatorname{gcd}(c, d) \neq 1$ then $c \mathbb{Z} \times d \mathbb{Z}$ has skew ideals.

Example 8. The principal ideal

$$
I(2,2)=(2,2) \mathbb{Z}+(4 \mathbb{Z} \times 4 \mathbb{Z})=(4 \mathbb{Z} \times 4 \mathbb{Z}) \cup((2+4 \mathbb{Z}) \times(2+4 \mathbb{Z}))
$$

of $2 \mathbb{Z} \times 2 \mathbb{Z}$ generated by $(2,2)$ is skew since $(0,0),(2,2) \in I(2,2)$, but $(0,2) \notin I(2,2)$. This is in accordance with Theorem 6 .

Using Theorem 2 we can generalize the situation described in Example 5. Namely, we can derive a Mal'cev type condition characterizing varieties of commutative rings whose ideals are directly decomposable.

Theorem 9. Let $\mathcal{V}$ be a variety of commutative rings. Then $\mathcal{V}$ has directly decomposable ideals if and only if there exists a unary term $t$ satisfying the identity $x t(x) \approx x$.

Proof. First assume $\mathcal{V}$ to have directly decomposable ideals. Consider the free commutative ring $\mathbf{F}(x)=(F(x),+, \cdot)$ with one free generator $x$ and the principal ideal

$$
\begin{aligned}
I(x, x) & =\{n(x, x)+(x, x)(r(x), s(x)) \mid n \in \mathbb{Z} \text { and }(r(x), s(x)) \in F(x) \times F(x)\}= \\
& =\{(n x+x r(x), n x+x s(x)) \mid n \in \mathbb{Z} \text { and } r(x), s(x) \in F(x)\}
\end{aligned}
$$

of $\mathbf{F}(x) \times \mathbf{F}(x)$ generated by $(x, x)$. Since $I(x, x)$ is directly decomposable and $(0,0)$, $(x, x) \in I(x, x)$, we have $(x, 0) \in I(x, x)$. Hence there exist some $n \in \mathbb{Z}$ and $r(x), s(x) \in$ $F(x)$ with $(n x+x r(x), n x+x s(x))=(x, 0)$ which implies $x t(x)=x$ with $t(x):=$ $r(x)-s(x)$. Conversely, assume there exists a unary term $t$ satisfying $x t(x) \approx x$. Let $\mathbf{R}_{1}=\left(R_{1},+, \cdot\right), \mathbf{R}_{2}=\left(R_{2},+, \cdot\right) \in \mathcal{V}$ and $I \in \operatorname{Id}\left(\mathbf{R}_{1} \times \mathbf{R}_{2}\right)$ and assume $(a, b) \in I$. Then $(a, 0)=(a, b)(t(a), 0) \in I$ and $(0, b)=(a, b)(0, t(b)) \in I$. According to (iii) of Theorem 2 , $I$ is directly decomposable.

Using Theorem 9, we can explicitly describe all varieties of commutative rings having directly decomposable ideals.

Corollary 10. A variety of commutative rings has directly decomposable ideals if and only if it satisfies an identity of the form $\sum_{i=2}^{n} a_{i} x^{i} \approx x$ with $n \geq 2$ and $a_{2}, \ldots, a_{n} \in \mathbb{Z}$. 
Proof. This follows immediately from Theorem 9 since the unary terms in a variety of

commutative rings are exactly the terms of the form $\sum_{i=1}^{n} a_{i} x^{i}$ with $n \geq 1$ and $a_{1}, \ldots, a_{n} \in$ $\mathbb{Z}$.

Corollary 11. The variety of Boolean rings has directly decomposable ideals since it satisfies the identity $x^{2} \approx x$.

However, we can also consider classes of commutative rings which do not form a variety. In this case we cannot apply Theorem 9 in order to prove that rings belonging to such a class have directly decomposable ideals. A typical example is the following:

Example 12. Let $\mathbf{R}=(R,+, \cdot)$ be a commutative ring, $\mathbf{F}=(F,+, \cdot)$ a field, $I \in$ $\operatorname{Id}(\mathbf{R} \times \mathbf{F})$ and $J:=\pi_{1}(I)$. If $I \subseteq R \times\{0\}$ then $I=J \times\{0\}$. Now assume $I \nsubseteq R \times\{0\}$. Then there exists some $(a, b) \in I$ with $b \neq 0$. If $(c, d) \in J \times F$ then there exists some $e \in F$ with $(c, e) \in I$ and hence

$$
(c, d)=(c, e)+(a, b)\left(0, b^{-1}(d-e)\right) \in I
$$

showing $I=J \times F$. Hence, $\mathbf{R} \times \mathbf{F}$ has directly decomposable ideals. By induction we obtain that $\mathbf{R} \times \mathbf{F}_{1} \times \cdots \times \mathbf{F}_{n}$ has directly decomposable ideals if $n \geq 1$ and $\mathbf{F}_{1}, \ldots, \mathbf{F}_{n}$ are fields.

In particular, we can consider the case where $\mathbf{R}$ denotes the commutative ring $\mathbf{K}$ from Example 1. Then $\mathbf{K} \times \mathbf{F}$ has directly decomposable ideals despite the fact that $\mathbf{K} \times \mathbf{K}$ does not have this property. Similarly, if $c$ and $d$ are integers satisfying $\operatorname{gcd}(c, d) \neq 1$ then $(c \mathbb{Z} \times d \mathbb{Z}) \times \mathbf{F}, c \mathbb{Z} \times \mathbf{F}$ and $d \mathbb{Z} \times \mathbf{F}$ have directly decomposable ideals though $c \mathbb{Z} \times d \mathbb{Z}$ does not have this property.

Remark 13. Note that Example 12 remains valid in case that $\mathbf{R}$ is not commutative.

Acknowledgement. The authors thank the referee for his valuable suggestions which increased the quality of the paper.

\section{References}

[1] I. T. Adamson, Rings, modules and algebras. Oliver and Boyd, Edinburgh 1971.

[2] I. Chajda and H. Länger, Commutative rings whose ideal lattices are complemented. Asian-European J. Math. (to appear).

[3] G. A. Fraser and A. Horn, Congruence relations in direct products. Proc. Amer. Math. Soc. 26 (1970), 390-394.

[4] J. Lambek, Lectures on rings and modules. Chelsea Publ. Co., New York 1976.

Authors' addresses:

Ivan Chajda

Palacký University Olomouc 
Faculty of Science

Department of Algebra and Geometry

17. listopadu 12

77146 Olomouc

Czech Republic

ivan.chajda@upol.cz

Günther Eigenthaler

TU Wien

Faculty of Mathematics and Geoinformation

Institute of Discrete Mathematics and Geometry

Wiedner Hauptstraße 8-10

1040 Vienna

Austria

guenther.eigenthaler@tuwien.ac.at

Helmut Länger

TU Wien

Faculty of Mathematics and Geoinformation

Institute of Discrete Mathematics and Geometry

Wiedner Hauptstraße 8-10

1040 Vienna

Austria, and

Palacký University Olomouc

Faculty of Science

Department of Algebra and Geometry

17. listopadu 12

77146 Olomouc

Czech Republic

helmut.laenger@tuwien.ac.at 